International Journal of Medical Sciences

ISSN 1449-1907 www.medsci.org 2008 5(4):197-202

Research Paper

(C) Ivyspring International Publisher. All rights reserved

\title{
BRCAl mutations in Algerian breast cancer patients: high frequency in young, sporadic cases
}

\author{
Nancy Uhrhammer ${ }^{1}$, Amina Abdelouahab ${ }^{2}$, Laurence Lafarge ${ }^{1}$, Viviane Feillel ${ }^{3}$, Ahmed Ben Dib $^{2}$, \\ Yves-Jean Bignon ${ }^{1}$
}

1. Laboratoire Diagnostic Génétique et Moléculaire, Centre Jean Perrin, 58 rue Montalembert, 63011 Clermont-Ferrand, France;

2. Service de Sénologie, Centre Pierre et Marie Curie, 1 Avenue Battendier, Algiers, Algeria;

3. Institut Claudius Regaud, 20 rue du Pont St Pierre, 31052 Toulouse, France.

Correspondence to: Yves-Jean Bignon, Laboratoire Diagnostic Génétique et Moléculaire, Centre Jean Perrin, 58 rue Montalembert, Clermont-Ferrand 63011, France. Tel: (33)473-27-80-50. Fax: (33)473-27-80-42. email: yves-jean.bignon@cjp.fr.

Received: 2008.06.27; Accepted: 2008.07.07; Published: 2008.07.08

Breast cancer rates and median age of onset differ between Western Europe and North Africa. In Western populations, 5 to $10 \%$ of breast cancer cases can be attributed to major genetic factors such as $B R C A 1$ and $B R C A 2$, while this attribution is not yet well defined among Africans. To help determine the contribution of $B R C A 1$ mutations to breast cancer in a North African population, we analysed genomic DNA from breast cancer cases ascertained in Algiers.

Both familial cases (at least three breast cancers in the same familial branch, or two with one bilateral or diagnosed before age 40) and sporadic cases less than 38 years of age were studied. Complete sequencing plus quantitative analysis of the BRCA1 gene was performed. $9.8 \%(5 / 51)$ of early-onset sporadic and $36.4 \%(4 / 11)$ of familial cases were found to be associated with BRCA1 mutations. This is in contrast $10.3 \%$ of French HBOC families exhibiting a $B R C A 1$ mutation. One mutation, c.798_799delTT, was observed in two Algerian families and in two families from Tunisia, suggesting a North African founder allele. Algerian non-BRCA1 tumors were of significantly higher grade than French non-BRCA tumors, and the age at diagnosis for Algerian familial cases was much younger than that for French non-BRCA familial cases. In conclusion, we observed a much higher frequency of BRCA1 mutations among young breast cancer patients than observed in Europe, suggesting biological differences and that the inclusion criterea for analysis in Western Europe may not be applicable for the Northern African population.

Key words: breast cancer, familial cancer syndromes, BRCA1 mutation

\section{Introduction}

$B R C A 1$ mutations are responsible for a significant proportion of hereditary breast and ovarian cancer (HBOC) families. BRCA1 is responsible for more than $50 \%$ of HBOC families with at least four cancer cases [1], and of $\sim 15 \%$ of families overall. In Western populations, an inherited mutation of this gene confers a lifetime risk of breast cancer of up to $80 \%$, with up to $40 \%$ of carriers developing breast cancer by the age of 50 [2]. Penetrance may be modified by other risk or protective genes or environmental factors, most notably reproductive history and diet. The effect of lifestyle on penetrance of BRCA mutations is significant, as studies of western populations show that carriers born after 1940 have much higher breast cancer incidence and earlier onset than carriers born before 1940 [3].

Studies of breast cancer in the Maghreb (including Morocco, Algeria, Tunisia, Lybia and Mauritania) have shown striking differences in breast cancer patterns. Age-standardized incidence per 100,000 for breast cancer in 2002 was 23.5 in Algeria versus 91.9 in France [4]. The size and grade of breast tumors in the Maghreb are increased, while the median age of onset (48) is more than ten years younger than the European/North American median of 61 [5]. About $11 \%$ of breast cancer cases in Algeria occur in women $\leq 35$ years old, and $55 \%$ of cases at $\leq$ 50 years. These differences may be due to differences in exposure to female hormones, diet, physical activity, or other factors. 
The combination of lower incidence and lower age of onset of breast cancer in the Maghreb suggests that the contribution of genetic factors such as mutation of BRCA1 may contribute to a larger proportion of breast cancer overall. We therefore set out to determine the contribution of mutations in the BRCA1 gene to breast cancer in Algeria. This was a pilot study to determine inclusion criterea for more widespread molecular diagnostic analysis of at-risk women in the Maghreb. We used the same criterea to define 'familial' cases that we use in our molecular diagnostic laboratory in France, and then added early-onset sporadic cases. All exons and splice-junctions of BRCA1 were amplified and sequenced. The MLPA method was used to detect larger deletions and duplications of the gene.

\section{Methods}

Breast cancer cases were identified at the Pierre and Marie Curie Hospital in Algiers and chosen according to the following criterea: age at diagnosis $\leq$ 38 years for sporadic cases; two or more first degree relatives with breast or ovarian cancer for familial cases. No families with ovarian cancer were ascertained. Approval was obtained from local institutional review boards, and each patient gave written informed consent. Peripheral blood was drawn from 51 early onset sporadic cases (average age at diagnosis $31.5 \pm 4.5$, range 15 to 38 ) and 13 cases from 11 breast cancer families (average age at diagnosis 37.2 \pm 6.8 , range 28 to 52 ).

DNA was extracted from $0.2 \mathrm{ml}$ of peripheral blood using the QIAamp DNA Blood Mini kit (Qiagen, Courtaboeuf, France). All exons and $\geq 50$ bp of each flanking intron were amplified in $15 \mu \mathrm{l}$ with 50ng DNA, 1x reaction buffer, $0.3 \mathrm{mM}$ dNTPs, $1 \mathrm{nM}$ primers, and 0.5 units Taq polymerase (primers from MWG Biotech, Ebersberg, Germany; all other reagents from Applied Biosystems, Courtaboeuf, France). Sequences available on request. PCR was performed in an MWG Bioblock thermocycler with initial denaturation at $94^{\circ} \mathrm{C}$ for $2 \mathrm{~min}$, followed by 30 to 35 cycles of $\left(94^{\circ} \mathrm{C} 20 \mathrm{~s}, 54^{\circ} \mathrm{C} 20 \mathrm{~s}, 72^{\circ} \mathrm{C} 20 \mathrm{~s}\right)$, except for exons $7\left(15\right.$ cycles of $94^{\circ} \mathrm{C} 20 \mathrm{~s}, 60^{\circ} \mathrm{C} 10 \mathrm{~s}, 72^{\circ} \mathrm{C} 20 \mathrm{~s}$ then 25 cycles of $\left.94^{\circ} \mathrm{C} 20 \mathrm{~s}, 56^{\circ} \mathrm{C} 15 \mathrm{~s}, 72^{\circ} \mathrm{C} 20 \mathrm{~s}\right)$ and $23(5$ cycles of $94^{\circ} \mathrm{C} 20 \mathrm{~s}, 57^{\circ} \mathrm{C} 20 \mathrm{~s}, 72^{\circ} \mathrm{C} 20$ s then 30 cycles of $94^{\circ} \mathrm{C} 20 \mathrm{~s}, 53^{\circ} \mathrm{C} 20 \mathrm{~s}, 72^{\circ} \mathrm{C} 20 \mathrm{~s}$ ). Exon 11 was analysed in nine overlapping PCR fragments. PCR products were purified by membrane retention (Multiscreen PCR, Millipore, Molsheim, France) and resuspended in $25 \mu \mathrm{l}$ of water; $3 \mu \mathrm{l}$ was then sequenced in a total of $8 \mu \mathrm{l}$ using $1 \mathrm{nM}$ primer and $3 \mu \mathrm{l}$ of Big Dye v3 reagents (Applied Biosystems, Courtaboeuf, France), purified over sephadex (Amersham Biosciences, Orsay,
France), $10 \mu \mathrm{l}$ of deionized formamide (Applied Biosystems, Courtaboeuf, France) added, and then resolved on a 3100 sequencer (Applied Biosystems, Courtaboeuf, France).

Sequences were compared to the BRCA1 genomic and cDNA reference sequences (Accession $\mathrm{N}^{\circ} \mathrm{s}$ L78833.1 and U14680 respectively) using Seqman software (Lasergene, Madison WI, USA). All mutations were confirmed on an independent second amplification and a second DNA sample where possible. Nucleotide numbering of all mutations and polymorphisms is in reference to the coding sequence, with the A of the initiating ATG $=1$.

Samples for which no point mutation was found were analysed by MLPA for large deletions or duplications according to the manufacturer's protocol (MRC Holland) (protocol available on request).

Haplotypes were determined at the following loci: D17S1321, D17S855, D17S1322, D17S1323, and D17S1327 (references and primer sequences NCBI) using PCR with fluorescent forward primers and analysis with Genescan software (Applied Biosystems, Courtaboeuf, France) as well as at biallelic polymorphisms in the coding sequence.

Statistical analysis used the chi-squared test, with $p \leq 0.05$ taken as the threshold for significant difference.

\section{Results}

Five deleterious mutations among the 51 early-onset sporadic cases were observed, and four mutations among the 11 families (Table 1). Two non-conservative missense variants, c.425C $>\mathrm{A}$ (p.Pro142His) and c.4072G>A (p.Gly1358Lys), and an intronic transversion with weak potential to affect splicing of exon 24, c.5467-10C >A (IVS23-10C >A), were observed in three sporadic cases: it is not currently known if these are deleterious mutations or rare polymorphisms. Other rare polymorphisms or conservative missense variants of unknown effect were also observed in some sporadic cases; none were predicted to affect splicing.

The c.798_799delTT mutation observed in families 1351 and 1612 was also observed in two families from Tunisia (data not shown). Microsatellite markers in and flanking the BRCA1 locus showed a common haplotype in all c.798_799delTT carriers.

Complete sequencing also provided data on snps in the coding sequence, allowing the construction of haplotypes. For a core of nine snps, 18 different haplotypes were observed for the 128 chromosomes studied (Table 2). The most common, observed 74 times, corresponded to the major canonical haplotype, H1, found by Judkins et al [6]. Additional snps 
dividing this canonical haplotype into three others were not informative in our study. Two other haplotypes described in that study of a North American / European population were also observed, $\mathrm{H} 7$ and H10, as well as a total of four copies of three haplotypes described in the Tunisian population [7]. The remaining 46 Algerian chromosomes carried 12 different haplotypes not described in either previous study; one of these was the second most frequent haplotype observed, at 18 copies. Six haplotypes were limited to single homozygous individuals. Quantitative analysis of BRCA1 exons did not suggest any large deletions that could confound homozygosity with hemizygosity.

There was no significant difference in the average age of mutated sporadic cases $(32.8 \pm 5.0$ years) versus non-mutated sporadic cases $(31.3 \pm 4.4$ years $)$, nor between familial mutated vs non-mutated cases (38.4 \pm
4.8 vs $35.7 \pm 8.9$ years, respectively). Algerian familial cases, regardless of BRCA status, were similar to French $B R C A 1$ cases $(37.2 \pm 6.3$ vs $41.2 \pm 10.4 ; \mathrm{p}=0.19)$, younger than French $B R \bar{C} A 2$ cases $(47.5 \pm 14.5 ; \mathrm{p}=$ 0.015 ) and much younger than French non-BRCA familial cases $(50.7 \pm 12, p=0.00017)$.

Tumor characteristics were compared between $B R C A 1$ heterozygotes and non-heterozygotes in the two populations (Table 3). All tumors of medullary histology were observed in BRCA1 heterozygotes. $B R C A 1$ tumors tended to be of higher grade in both populations, as expected; however, Algerian non-BRCA1 cases included a significant excess of high grade tumors $(p<0.001)$. Significantly more Algerian non-BRCA1 tumors were ER-negative and node-positive, compared to French non-BRCA1 tumors $(\mathrm{p}<0.001$ and $\mathrm{p}<0.002$, respectively), consistent with their higher grade tumors.

Table 1. Characteristics of patients with BRCA1 mutations or unclassified variants.

\begin{tabular}{|c|c|c|c|c|c|c|c|c|c|c|c|c|c|c|}
\hline Case & mutation & effect & $\begin{array}{l}\text { Sporadic } \\
\text { or fa- } \\
\text { milial }\end{array}$ & $\begin{array}{l}\text { Age at } \\
\text { diagnosis }\end{array}$ & histology & size & grade & ER & PR & nodes & $\begin{array}{l}\text { Age at } \\
\text { menarche }\end{array}$ & parity & nursing & BMI \\
\hline 1357-01 & c.46_74del29 & p.Asn16fs & Sporadic & 29 & Papillary & $4 \mathrm{~cm}$ & III & - & - & n.i & 14 & 0 & 0 & 21.3 \\
\hline 1490-01 & c.46_74del29 & p.Asn16fs & Familial & $37+44$ & $\begin{array}{l}\text { atypical medullar, } \\
\text { atypical ductal }\end{array}$ & $3 \mathrm{~cm}$. & -- & n.i. & n.i & $0 / 1$ & n.i & n.i & n.i & 34.2 \\
\hline 1358-01 & c.83_84delTG & p.Arg28fs & Sporadic & 26 & $\begin{array}{l}\text { Poorly differentiated } \\
\text { ductal }\end{array}$ & $1 \mathrm{~cm}$ & II & - & - & $0 / 11$ & 12 & 0 & 0 & 20.3 \\
\hline $1497-01$ & c. $202+1 G>A$ & $\begin{array}{l}\text { Splice do- } \\
\text { nor }\end{array}$ & Familial & 38 & $\begin{array}{l}\text { Atypical infiltrating } \\
\text { ductal }\end{array}$ & $9 \mathrm{~cm}$ & III & + & + & $1 / 14$ & n.i & n.i & n.i & n.i \\
\hline 1497-02 & & exon 5 & & 42 & $\begin{array}{l}\text { polymorphic infiltrating } \\
\text { ductal }\end{array}$ & $\begin{array}{l}3.5 \\
\mathrm{~cm}\end{array}$ & III & - & - & $3 / 10$ & 15 & 6 & $54 \mathrm{~m}$ & 22.7 \\
\hline $1612-01$ & c.798_799delTT & p.Val266fs & Familial & 43 & $\begin{array}{l}\text { polymorphic infiltrating } \\
\text { ductal }\end{array}$ & $\begin{array}{l}0.7 \\
\mathrm{~cm}\end{array}$ & II & n.i. & n.i & $0 / 12$ & 15 & 3 & $72 \mathrm{~m}$ & 21.8 \\
\hline 1351-01 & c.798_799delTT & p.Val266fs & Familial & 32 & infiltrating ductal & $6 \mathrm{~cm}$ & II & n.i. & n.i & $11 / 20$ & 12 & 0 & 0 & 21.6 \\
\hline 1351-02 & & & & 33 & n.i. & & & & & & & & & \\
\hline 1370-01 & c.1817delC & p.Pro606fs & Sporadic & 37 & $\begin{array}{l}\text { atypical infiltrating duc- } \\
\text { tal }\end{array}$ & $4 \mathrm{~cm}$ & III & n.i. & - & $4 / 11$ & 12 & 0 & 0 & 26.0 \\
\hline 1614-01 & c.2745dupT & p.Ser915fs & Sporadic & 36 & Sarcomatoid carcinoma & $5 \mathrm{~cm}$ & III & n.i. & - & $1 / 10$ & 12 & 6 & $36 \mathrm{~m}$ & \\
\hline $1470-01$ & c.3715delT & p.Ser1239fs & Sporadic & 36 & Infiltrating & $\begin{array}{l}3.5 \\
\mathrm{~cm}\end{array}$ & II & n.i. & n.i & $0 / 18$ & 12 & 3 & $72 \mathrm{~m}$ & 22.6 \\
\hline \multicolumn{15}{|c|}{ Samples with unclassified variants that may be involved in breast cancer } \\
\hline $1620-01$ & c. $425 \mathrm{C}>\mathrm{A}$ & Pro142His & Sporadic & 26 & infiltrating ductal & $\begin{array}{l}1.5 \\
\mathrm{~cm}\end{array}$ & III & n.i. & + & $14 / 18$ & 14 & 0 & 0 & 23.9 \\
\hline 1355-01 & c. $4072 \mathrm{G}>\mathrm{A}$ & Glu1358Lys & Sporadic & 35 & $\begin{array}{l}\text { infiltrating ductal + in } \\
\text { situ }\end{array}$ & n.i. & n.i. & n.i. & n.i. & n.i. & n.i. & n.i. & n.i. & n.i. \\
\hline 1468-01 & Ivs23-10C $>A$ & $* *$ & Sporadic & 28 & Atypical ductal & $3 \mathrm{~cm}$ & III & n.i. & n.i. & $1 / 1$ & 12 & 0 & 0 & 20.9 \\
\hline \multicolumn{15}{|c|}{ Samples with unclassified variants that are not likely to be involved in breast cancer } \\
\hline \multirow[t]{3}{*}{$1476-01$} & c. $981 \mathrm{~A}>\mathrm{G}$ & Thr327Thr & Sporadic & 34 & Polymorphic ductal & 0.3 & II & n.i. & + & $1 / 21$ & 12 & 0 & 0 & 28.6 \\
\hline & c. $2733 \mathrm{G}>\mathrm{A}$ & Gly911Gly & & & & & & & & & & & & \\
\hline & c. $3024 \mathrm{G}>\mathrm{A}$ & Met1008Ile & & & & & & & & & & & & \\
\hline \multirow[t]{3}{*}{ 1494-01 } & c. $981 \mathrm{~A}>\mathrm{G}$ & Thr327Thr & Sporadic & 37 & infiltrating galactophoric & $7 \mathrm{~cm}$ & II & n.i. & n.i. & $0 / 1$ & 13 & 2 & 0 & 21.0 \\
\hline & c. $2733 A>G$ & Gly911Gly & & & & & & & & & & & & \\
\hline & c. $3024 \mathrm{G}>\mathrm{A}$ & Met1008Ile & & & & & & & & & & & & \\
\hline 1493-01 & c. $4883 \mathrm{~T}>\mathrm{C}$ & Met1628Thr & Sporadic & $38(?)$ & n.i. & n.i. & n.i & n.i. & n.i. & n.i & n.i. & n.i. & n.i & n.i \\
\hline 1488-01 & c. $4956 \mathrm{G}>\mathrm{A}$ & Met1652Ile & Sporadic & 26 & infiltrating ductal & $\begin{array}{l}2.5 \\
\mathrm{~cm}\end{array}$ & II & + & - & pos & n.i. & n.i. & n.i. & n.i. \\
\hline 1480-01 & c. $5117 \mathrm{G}>\mathrm{C}$ & Gly1706Ala & Sporadic & 36 & $\begin{array}{l}\text { infiltrating ductal }+ \text { in } \\
\text { situ }\end{array}$ & $2 \mathrm{~cm}$ & n.i. & n.i. & n.i & $1 / 10$ & n.i. & n.i. & n.i. & n.i. \\
\hline 1610-01 & c. $5117 \mathrm{G}>\mathrm{C}$ & Gly1706Ala & Familial & 29 & infiltrating ductal & $5 \mathrm{~cm}$ & III & - & + & $3 / 26$ & 13 & 2 & $6 \mathrm{~m}$ & 27.3 \\
\hline 1362-01 & c. $5175 \mathrm{~A}>\mathrm{G}$ & Glu1725Glu & Sporadic & 32 & Micro-infiltrating ductal & $\begin{array}{l}5.5 \\
\mathrm{~cm}\end{array}$ & II & n.i. & n.i. & $0 / 13$ & 12 & 3 & $16 \mathrm{~m}$ & 22.8 \\
\hline
\end{tabular}


Table 2. BRCA1 Haplotypes among Algerian breast cancer patients

\begin{tabular}{|c|c|c|c|c|c|c|c|c|c|c|c|c|c|c|c|c|c|c|}
\hline Snp \Haplotype & H1 & H7 & $\mathrm{H} 10$ & $\mathrm{~T} 2$ & $\mathrm{~T} 4$ & T17 & A1 & A2 & A3 & $\mathrm{A} 4$ & A5 & A6 & A7 & A8 & A9 & A10 & A11 & A12 \\
\hline c. $2077 \mathrm{~A}$ & 0 & 0 & 0 & 0 & 0 & 0 & 0 & 0 & 0 & 0 & 0 & 0 & 1 & 1 & 1 & 0 & 0 & 0 \\
\hline c. $2082 \mathrm{~T}$ & 0 & 0 & 0 & 0 & 0 & 0 & 0 & 0 & 0 & 1 & 1 & 1 & 1 & 1 & 1 & 1 & 1 & 1 \\
\hline c. $2311 \mathrm{C}$ & 0 & 0 & 0 & 0 & 0 & 0 & 0 & 1 & 1 & 1 & 1 & 1 & 0 & 1 & 1 & 1 & 0 & 1 \\
\hline c. $2521 \mathrm{C}$ & 0 & 0 & 1 & 1 & 0 & 0 & 0 & 0 & 0 & 0 & 0 & 0 & 0 & 0 & 0 & 0 & 0 & 0 \\
\hline c.3113G & 0 & 0 & 0 & 0 & 0 & 0 & 0 & 1 & 1 & 1 & 1 & 1 & 1 & 1 & 1 & 1 & 0 & 1 \\
\hline c.3119G & 0 & 1 & 0 & 0 & 0 & 0 & 0 & 0 & 0 & 0 & 0 & 0 & 0 & 0 & 0 & 0 & 1 & 0 \\
\hline c. $3548 \mathrm{G}$ & 0 & 0 & 0 & 0 & 1 & 0 & 1 & 1 & 1 & 0 & 1 & 1 & 1 & 1 & 1 & 1 & 0 & 1 \\
\hline c. $4308 \mathrm{C}$ & 0 & 0 & 0 & 0 & 0 & 0 & 1 & 1 & 1 & 1 & 1 & 1 & 1 & 1 & 1 & 1 & 1 & 0 \\
\hline c. $4837 \mathrm{G}$ & 0 & 0 & 0 & 1 & 0 & 1 & 0 & 0 & 1 & 0 & 0 & 1 & 0 & 1 & 0 & 0 & 1 & 0 \\
\hline Times appearing & 74 & 4 & 2 & 1 & 2 & 1 & 2 & 2 & 2 & 2 & 5 & 18 & 2 & 7 & 1 & 1 & 1 & 1 \\
\hline \multirow{2}{*}{$\%$ of 128 alleles } & 57.8 & 3.1 & 1.5 & 0.8 & 1.5 & 0.8 & 1.5 & 1.5 & 1.5 & 1.5 & 3.9 & 14 & 1.5 & 5.5 & 0.8 & 0.8 & 0.8 & 0.8 \\
\hline & & & & & * & & * & * & * & * & & & * & & & & & \\
\hline
\end{tabular}

H1, H7 and H10 are described by Judkins et al [6]; T2, T4 and T17 correspond to haplotypes described by Troudi et al [7] ; A1 through A12 were unique to the Algerian population. 0 indicates the nucleotide corresponding to the reference sequence ; 1 to the variant nucleotide. Asterisk indicates a haplotype unique to a homozygous individual.

Table 3. Comparison of tumor characteristics from mutated and non-mutated cases, from Algeria and France.

\begin{tabular}{lllll}
\hline & BRCA1, Algiers & Non-BRCA1, Algiers & BRCA1, France & Non-BRCA, France* \\
Age at diagnosis & $36.1 \pm 5.5^{* *}$ & $31.7 \pm 5.4^{* * *}$ & $41.2 \pm 10.4$ & $50.7 \pm 12.2$ \\
\hline Grade 1 & 1 of $10(10 \%)$ & 1 of $43(2 \%)$ & 2 of $39(5 \%)$ & 32 of $115(28 \%)$ \\
Grade 2 & 3 of $10(30 \%)$ & 25 of $43(58 \%)$ & 10 of $39(26 \%)$ & 67 of $115(58 \%)$ \\
Grade 3 & 6 of $10(60 \%)$ & 17 of $43(40 \%)$ & 27 of $39(69 \%)$ & 16 of $115(14 \%)$ \\
\hline Size & $3.8 \mathrm{~cm}$ & $3.4 \mathrm{~cm}$ & $2.0 \mathrm{~cm}$ & $2.2 \mathrm{~cm}$ \\
Medullary histology & 1 of 12 & 0 of 48 & 6 of 49 & 0 of 139 \\
ER, pos/tested & $1 / 5(20 \%)$ & $3 / 15(20 \%)$ & $5 / 14(36 \%)$ & $45 / 63(71 \%)$ \\
PR, pos/tested & $2 / 6(33 \%)$ & $18 / 32(56 \%)$ & $4 / 14(29 \%)$ & $35 / 64(55 \%)$ \\
Node positive & 6 of $10(60 \%)$ & 29 of $42(69 \%)$ & 13 of $31(42 \%)$ & 41 of $102(40 \%)$ \\
\hline
\end{tabular}

* non-BRCA cases were found to be negative for mutations in both BRCA1 and BRCA2; ** includes 5 cases selected for age $\leq 38$ years; *** includes $46 / 52$ cases selected for age $\leq 38$ years.

\section{Discussion}

The types of BRCA1 mutations in this sample of the Algerian population were typical of those observed elsewhere, with six different deletions or duplications involving one to 29 nucleotides, and a novel change in the donor splice site of exon 5. Two nonconservative substitutions of amino acids were observed, as well as an intronic transversion with some potential to affect splicing of exon 24, possibly representing novel mutations in this population. Several uncommon silent or conservative sequence variants were also observed.

We observed one deleterious mutation, c.798_799delTT, in two Algerian families and also in two Tunisian breast cancer families (data not shown), suggesting the first non-Jewish founder mutation to be described in Northern Africa. This mutation is cited twice in the BIC database, without any ethnic origin indicated. Analysis of five microsatellite markers showed a common haplotype associated with this mutation in all our known carriers. None of the founder mutations previously observed among middle eastern (Iranian) or Jewish populations were found.

Haplotype analysis revealed the genetic diversity of the Algerian population. A large study of North Americans and European revealed 10 canonical haplotypes clustered around two major haplotypes both diverged from a common ancestor [6]. Analysis of the Tunisian population revealed several new haplotypes, in concordance with the great age of this population [7]. In keeping with this, the Algerian population also exhibited several unique haplotypes as well as three in common with the Tunisian population. Three haplotypes could be considered 'common', accounting for 58, 14 and $5.5 \%$ of observed chromosomes. All the Algerian haplotypes appeared to be derived from the major $\mathrm{H} 1$ chromosome described by both Judkins and Troudi; none appeared related to the other major haplotype, H2. Interestingly, several rare haplotypes occurred as homozygotes. Although we have no information on the precise geographic or tribal origin of the families, we speculate that this may reflect the insular nature of rural Algeria, where the coefficient of inbreeding is relatively high and genetic drift may establish unique regional haplotypes. No homozygosity for unclassified variants was observed.

The age at which familial Algerian cases, regardless of $B R C A 1$ status, developed breast cancer was similar to our BRCA1-positive French families, but significantly younger than French familial non-BRCA or BRCA2 cases. Young age at diagnosis is an indication for referral for BRCA testing, and the older age at cancer in BRCA-negative families is common. 
That familial cases from the Maghreb without BRCA1 mutation resemble $B R C A 1$ families may be related to the lower age of onset and higher frequency of high grade tumors overall for breast cancer in this population.

The characteristics of $B R C A 1$-related tumors were similar between Algerian and French patients, allowing for larger tumor size probably associated with later diagnosis of Algerian cases. In contrast, the non-BRCA1 tumors from Algeria were also of significantly higher grade, presented more positive nodes and were less frequently ER-positive than French non-BRCA tumors. This excess of high-grade tumors in African populations has been described before, with 65 to $86 \%$ of tumors being grade II or III [8-10]. Frequently positive nodes and negative hormone receptor status are both consistent with high-grade tumors. Low- and high-grade breast cancers may represent separate pathways of oncogenesis [11], thus the absence of low grade tumors is not explained by delay in diagnosis allowing 'progression' to a higher grade. The marked difference in distribution of breast tumor grades between Western and Middle-Eastern/African societies merits further study. One possibility is that low-grade tumors either arise infrequently or do arise but don't develop into palpable tumors initiating medical care. This relative absence of low-grade tumors may contribute to the lower incidence of breast cancer overall in African countries. It may also reflect an ascertainment bias: Western societies have instituted widespread screening programs detecting small low-grade tumors that may go undeclared in developing societies. Two arguments for the biological basis of this difference have been proposed. First, migrants from low-incidence countries gradually take on some of the risk of breast cancer of their host countries, and their descendants have a risk of breast cancer corresponding to the host country $[12,13]$, arguing for environmental and lifestyle factors in the difference in incidence. On the other hand, studies in the United States have shown that breast cancer in African-American women is associated with higher grade and poorer prognosis, arguing a biological difference even after socio-economic differences are controlled, and reflecting breast cancer statistics for sub-Saharan Africa [14].

Environmental and lifestyle factors may be largely responsible for the low incidence of breast cancer in the Maghreb. These factors are difficult to identify precisely, but their combined effect has serious consequences, as the clear increase in breast cancer incidence in American Ashkenazi BRCA carriers born after vs before 1940 shows [3].
Protective reproductive factors tend to diminish as societies become "westernized" and women delay and limit their families. The protective effect of pregnancy is associated with younger age at first pregnancy as well as with increasing parity, while longer breastfeeding has an independent protective effect $[15,16]$. Parity levels are converging for Europe and the Maghreb, with 6.49 children per woman in Algeria and 1.87 in France in 1980-1985, but 2.53 in Algeria and 1.87 in France in 2000-2005 (http:/ / www.un.org/esa/population/ordering.htm). In the present study, parity was lower among women from Algeria $(1.84 \pm 2.04)$ than from France $(2.18 \pm$ $1.52)$, probably because many of the women in our study were of childbearing age and had not completed their families (whereas there are many more older carriers in our french families).

It thus seems that a major protective factor in the Algerian population is rapidly disappearing; the reduction in parity is likely accompanied by increased age at first pregnancy and reduced duration of breastfeeding. This change in lifestyle may soon be reflected in increased breast cancer incidence as this cohort of women reaches the age at which breast cancer is most prevalent. Other lifestyle factors that may have contributed to breast cancer risk in western populations for multiple generations now but which have only more recently begun to affect the Magrheb include the use of oral contraceptives, less physical activity, increased use of refined foods and chemical food additives, and decreased intake of fresh fruits and vegetables.

Single cases are not generally accepted for genetic testing for hereditary breast cancer genes without a strong implication of hereditary factors, such as young age at diagnosis ( $\leq 35$ years), multifocal or bilateral tumors, and/or medullar histology. In most western populations such testing is not cost-effective, with only $2.6 \%$ of 2-case families in Finland being positive for a BRCA mutation [17], and very few sporadic cases being positive in the US. Other studies, however, suggest that testing of 2-case families or single cases before age 36 can be efficient in certain populations [18, 19]. The $9.8 \%$ BRCA1 mutation frequency we observed in young sporadic cases in Algeria is remarkable in comparison to these other populations. At least two explanations may contribute to this observation: the misclassification of familial cases, and a different population structure in Algeria, with a relatively low incidence of breast cancer revealing the greater contribution of genetic factors.

Although our sporadic cases did not signal any family history of breast or ovarian cancer, the stigma attached to cancer in this society makes it is possible 
that they were not aware of a positive history. Our discussions with familial cases showed that women with breast cancer often hid this diagnosis from their close relatives. At this time, the medical structures in place, such as cancer registries, are not sufficient to ascertain family history other than by asking the index case.

The second hypothesis, that of the relatively greater contribution of genetic factors in a population where the overall incidence of breast cancer is low, would suggest a greater proportion of familial vs sporadic cases. Although we have not yet performed a population-based study to determine this ratio, the high frequency of BRCA1 mutation in isolated cases may indicate that this is the case, especially if the penetrance of BRCA1 mutations is lower in this population. The protective lifestyle factors discussed above may have spared the relatives of our young isolated carriers from breast cancer in spite of their carrier status. Thus the western BRCA1 model where most mutations manifest in familial aggregations of breast and/or ovarian cancer with penetrance for breast cancer of $50 \%$ by age 50, may be expressed differently in the Maghreb, where mutations are found in familial cancer but also in a significant proportion of isolated cases, penetrance is reduced. It remains to be seen whether recent changes in lifestyle will increase the incidence of breast cancer in carrier families.

In conclusion, our findings suggest that the norms of accepting breast cancer cases for BRCA analysis must be adapted to the population. We are extending our study to additional cases and families from Algeria, and hope to soon be able to compare these results with our analyses of the Tunisian, Lebanese and Moroccan populations. The role of $B R C A 2$ in breast cancer in the Maghreb is also under study.

\section{Acknowledgements}

We would like to thank the patients participating in this study, as well as F. Kwiatkowski for his help with the statistical analysis and critical review of the manuscript. This work was funded by the Ministère de Santé in France and the Pierre and Marie Curie Institute in Algeria.

\section{Conflict of Interest}

The authors have declared that no conflict of interest exists.

\section{References}

1. Ford D, Easton D, Bishop D, et al. Risks of cancer in BRCA1-mutation carriers. Breast Cancer Linkage Consortium. Lancet, 1994, 343(8899): 692-695
2. Antoniou A, Pharoah $\mathrm{P}, \mathrm{Narod} \mathrm{S}$, et al. Average risks of breast and ovarian cancer associated with BRCA1 or BRCA2 mutations detected in case Series unselected for family history: a combined analysis of 22 studies. Am J Hum Genet, 2003, 72(5): 1117-1130.

3. King MC, Marks JH, Mandell JB, et al. Breast and ovarian cancer risks due to inherited mutations in BRCA1 and BRCA2. Science, 2003, 302(5645): 643-646.

4. [Internet] Globocan. http://www-dep.iarc.fr/globocan/data base.htm.

5. [Internet] Caducee. http://www.caducee.net/

6. Judkins T, Hendrickson BC, Deffenbaugh AM, et al. Application of Embryonic Lethal or Other Obvious Phenotypes to Characterize the Clinical Significance of Genetic Variants Found in Trans with Known Deleterious Mutations. Cancer Res, 2005, 65: 10096-10103.

7. Troudi W, Uhrhammer N, Sibille C, et al. Contribution of the BRCA1 and BRCA2 mutations to breast cancer in Tunisia. J Hum Genet, 2007, 52(11): 915-920.

8. Ben Ahmed S, Aloulou S, Bibi M, et al. Breast cancer prognosis in Tunisian women: analysis of a hospital series of 729 patients. Sante Publique, 2002, 14(3): 231-241.

9. Al-Kuraya K, Schraml P, Sheik S, et al. Predominance of high-grade pathway in breast cancer development of Middle East women. Mod Pathol, 2005, 18: 891-897.

10. Gukas ID, Jennings BA, Mandong BM, et al. Clinicopathological features and molecular markers of breast cancer in Jos, Nigeria. West Afr J Med, 2005, 24(3): 209-213.

11. Simpson PT, Reis-Filho J, Gale T, Lahani SR. Molecular evolution of breast cancer. J Pathol, 2005, 205: 248-254.

12. John EM, Phipps AI, Davis A, Koo J. Migration history, acculturation, and breast cancer risk in Hispanic women. Cancer Epidemiol Biomarkers Prev, 2005, 14(12): 2905-2913.

13. Yavari P, Hislop TG, Bajdik C, et al. Comparison of cancer incidence in Iran and Iranian immigrants to British Columbia, Canada. Asian Pac J Cancer Prev, 2006, 7(1): 86-90.

14. Fregene A, Newman L. Breast cancer in sub-Saharan Africa: how does it relate to breast cancer in African-American women? Cancer, 2005, 103(8): 1540-1550.

15. Collaborative Group on Hormonal Factors in Breast Cancer. Breast cancer and breastfeeding: collaborative reanalysis of individual data from 47 epidemiological studies in 30 countries, including 50302 women with breast cancer and 96973 women without the disease. Lancet, 2002, 360(9328): 187-195.

16. Bernier MO, Plu-Bureau G, Bossard N, Ayzac L, Thalabard JC. Breastfeeding and risk of breast cancer: a metaanalysis of published studies. Hum Reprod Update, 2000, 6(4): 374-386.

17. Vahteristo P, Eerola H, Tamminen A, Blomqvist C, Nevanlinna H. A probability model for predicting BRCA1 and BRCA2 mutations in breast and breast-ovarian cancer families. Br J Cancer, 2001, 84(5): 704-708.

18. Goelen G, Teugels E, Bonduelle M, Neyns B, De Greve J. High frequency of BRCA1/2 germline mutations in 42 Belgian families with a small number of symptomatic subjects. J Med Genet, 1999, 36(4): 304-308.

19. Peto J, Collins N, Barfoot R, et al. Prevalence of BRCA1 and BRCA2 gene mutations in patients with early-onset breast cancer. J Natl Cancer Inst, 1999, 91(11): 943-949. 